\title{
Estilo de vida, senso de controle e qualidade de vida: um estudo com a população idosa de Patos de Minas- MG
}

\section{Life style, perceived control and quality of life: a study with the aged population of Patos de Minas-MG}

\author{
Lourine Severo Oliveira* \\ Centro Universitário de Patos de Minas - UNIPAM, Patos de Minas, Minas Gerais, \\ Brasil
}

\section{Dóris Firmino Rabelo**}

Universidade Federal do Recôncavo da Bahia - UFRB, Cruz das Almas, Bahia, Brasil

\author{
Nelma Caires Queroz*** \\ Centro Universitário de Patos de Minas - UNIPAM, Patos de Minas, Minas Gerais, \\ Brasil
}

\begin{abstract}
RESUMO
O aumento populacional de idosos gerou uma preocupação e uma necessidade de repensar políticas e práticas relacionadas à velhice, demandando assim o desenvolvimento de pesquisas que investiguem as variáveis físicas e psicológicas a fim de proporcionar uma melhor qualidade de vida aos idosos. O objetivo da pesquisa foi identificar os comportamentos de saúde, o senso de controle e a qualidade de vida dos idosos residentes no município de Patos de Minas - MG. Participaram do estudo 364 idosos com 60 anos ou mais, não institucionalizados e que atingiram o escore mínimo esperado no Mini-exame do Estado Mental. Os resultados encontrados apontaram que a amostra apresenta um bom conjunto de comportamentos de saúde, são em grande maioria independentes tendo autonomia sobre si, apresentaram ter crenças de controle pessoal e interpessoal sobre como lidar com circunstâncias importantes de sua vida e avaliaram a qualidade de vida satisfatoriamente.
\end{abstract}

Palavras-chave: Senso de controle, Qualidade de vida, Idoso.

\section{ABSTRACT}

The increased population of aged generated a concern and a necessity to rethink practical politics and related to the oldness, thus demanding the development of research that investigates the physical and psychological variables in order to provide better quality of life for the aged ones. The aim was to investigate health behaviors, perceived control and the quality of life of the aged residents in the city of Patos de Minas - MG. Participated 364 aged, not institutionalized and that had reached the minimum score in the Mini-examination of the Mental State. The joined results had pointed that the sample presents a good set of health behaviors, the majority 
independent and having autonomy on itself, presented beliefs of personal and interpersonal control to deal with important circumstances of its life and had evaluated the quality of life satisfactorily.

Keywords: Perceived control, Quality of life, Elderly.

\section{I ntrodução}

A qualidade de vida na velhice ganhou relevância nos últimos 30 anos com a consciência do envelhecimento populacional. É um conceito complexo e multidimensional o que implica em várias definições aplicáveis não só a velhice, mas em qualquer outro momento do desenvolvimento humano (NERI, 2005a).

A Organização Mundial de Saúde (OMS), na década de 1990, definiu qualidade de vida como a percepção do indivíduo quanto à sua posição na vida, no contexto cultural e no sistema de valores em que vive, levando em conta, metas, expectativas, padrões e preocupações (FLECK; LOUSADA; XAVIER; CHACHAMOVICH; VIEIRA; SANTOS; PINZON, 1999). A partir de então o termo qualidade de vida deixou de estar associado à ausência de doença, passando a depender da interação entre indivíduo e meio, ambos em transformações constantes.

$\mathrm{Na}$ psicologia, dentre os critérios considerados para a avaliação da qualidade de vida na velhice, são utilizados o senso de controle, o senso de autoeficácia, o senso de significado, a competência social e cognitiva, e o bem-estar subjetivo (NERI, 2005b). Uma velhice bem sucedida depende, em grande, parte das crenças sobre as capacidades para manejar a própria vida e o mundo externo, já que nessa fase ocorrem perdas físicas, sensoriais, intelectuais e sociais significativas. Ser capaz de permanecer no controle de uma situação, seja ela grande ou pequena, aumenta as possibilidades de manter o idoso ativo e o ajuda a lidar com essas perdas (WROSCH; SCHULZ, 2008).

Dessa forma, acredita-se que senso de controle é uma percepção, de poder pessoal sobre o controle, o estabelecimento, e a direção para resultados e objetivos de vida. Segundo Silva e Yu (2009), na ausência do senso de controle, as pessoas tendem a se sentir inseguras, facilitando o surgimento de efeitos sobre seu desempenho, no exercício de papéis na sociedade, na convivência familiar, ou mesmo no desenvolvimento de suas atividades profissionais. O senso de controle permite compreender como as pessoas podem decidir sobre o que afeta ou poderá afetar a manutenção do seu estilo de vida. 
Para Ribeiro e Yassuda (2007), o estilo de vida pode ser entendido como a escolha dos alimentos e das atividades ocupacionais, sociais e físicas que influenciam a capacidade de evitar doenças, bem como, o nível de funcionamento físico e mental e o engajamento do sujeito com a vida. Segundo Straub (2005), é difícil imaginar uma atividade ou comportamento que não influencie a saúde de alguma forma. Certos comportamentos relacionados à saúde podem ter impacto positivo ou negativo. Sendo assim, os comportamentos ou estilos de vida e as crenças de controle de uma pessoa são capazes de influenciar sua saúde e sua qualidade de vida.

\subsection{Estilo de vida}

As escolhas de estilo de vida são moduladas pelo contexto socioeconômico no qual o individuo está inserido. Dessa forma, entende-se que promoção da saúde e a prevenção de doenças, conseguem-se não apenas com medidas médicas, mas também com ações sociopolíticas (RIBEIRO; YASSUDA, 2007).

Para Neri (2006a), no decorrer do ciclo de vida, o estilo de vida pode contribuir ou prejudicar a qualidade de vida. Nesse contexto, fazem parte a prevenção e os comportamentos pró-ativos em relação à preservação da saúde, por meio dos quais as pessoas exercem controle sobre a vitalidade e sobre a qualidade de sua saúde.

Petroski e Pelegrini (2009) definem o construto estilo de vida como um padrão de comportamento que pode ter profundo efeito na saúde dos seres humanos e está relacionado aos aspectos que refletem as atitudes, os valores e as oportunidades na vida das pessoas. Straub (2005) apresenta uma classificação sobre os comportamentos que influenciam de alguma forma a saúde como: patógenos comportamentais para aqueles comportamentos que prejudicam a saúde, e imunógenos comportamentais para aqueles comportamentos que apresentam fator protetor à saúde. Esses comportamentos estão constantemente inter-relacionados.

$\mathrm{O}$ estilo de vida adotado pelas pessoas determina a qualidade de seu envelhecimento, pois a escolha dos alimentos e do tempo gasto com atividades pessoais, sociais e atividades físicas exerce influência sobre o funcionamento físico, mental e também no engajamento do indivíduo com a vida (OLIVEIRA; DUARTE; LEBRÃO; RUY, 2007). Dessa forma, o estilo de vida pode ser compreendido como um conjunto de decisões individuais que afetam a saúde de alguma forma e sobre o qual se pode manter certo grau de controle. A esse grau de controle, ou a essa capacidade que temos de poder manter certo controle sobre as situações da vida chama-se senso de controle.

\subsection{Senso de Controle}


Para Skaff (2007) o senso de controle é uma tendência ao agir e ao sentir-se como alguém que pode influenciar várias situações da vida e exercer influência definitiva nos acontecimentos, seja por habilidades, desejo, imaginação ou escolha. O senso de controle é uma percepção de poder pessoal sobre o controle, o estabelecimento, e a direção para resultados e objetivos de vida de um indivíduo.

Nos anos 70, o senso de controle foi uma variável amplamente estudada, pois se acreditava que esta variável mediava a satisfação sobre 0 bem estar percebido. Pesquisas em psicologia do envelhecimento mostram que, o senso de controle é importante para o bem estar subjetivo em todas as idades. Para Silva e Yu (2009) pessoas com baixo senso de controle tendem a se sentir com pouco poder, acreditando que as oportunidades na vida são resultados de fatalidades, que os eventos são resultados da sorte, e ainda que pouco se possa fazer para controlar coisas ruins que ocorreram em suas vidas.

Os idosos podem ter seu bem estar subjetivo e sua funcionalidade afetada pelo senso de controle. Isso ocorre em decorrência das perdas significativas de saúde física e de limitações de recursos pessoais. No entanto, para Goldstein (2000), muitos idosos conseguem preservar um senso de eficácia e de controle sobre seu desenvolvimento. Acredita-se que há vários tipos de eventos que influenciam o senso de controle em idosos, e podem fazer deles pessoas vulneráveis ou mais resistentes. Entre esses eventos podemse destacar o contexto social, os eventos de vida e as características pessoais.

O contexto social diz respeito aos estereótipos sobre a velhice, segundo os quais todos os idosos são dependentes e confusos, e apresentam problemas de memória. Estes estereótipos levam parentes e cuidadores a ignorar os esforços do idoso, ou até mesmo pressionar para que o idoso produza comportamentos convenientes para os cuidadores, contribuindo para que os mesmos se desmotivem. Os eventos de vida são os acontecimentos na vida não previstos e não desejáveis, como doenças, morte do cônjuge, problemas dos filhos e dos netos. Por último, as características pessoais expressas por comportamentos perfeccionistas, aliados ao baixo senso de autoeficácia, podem fazer com que o idoso deixe de se comportar, por acreditar que é inútil e que não vai gerar as consequências desejadas.

O controle pessoal tem relação com a adoção e a manutenção de hábitos de vida saudável, com a evitação de riscos e com práticas preventivas, e contribuem para a continuidade do desempenho físico e mental, ou seja, agem como facilitadores da qualidade de vida na velhice. Em geral, qualidade de vida na velhice está associada com autonomia, saúde física, desempenho de atividades e papéis sociais além de uma vida com significado (NERI, 2005c). Contudo, o 
processo de envelhecer é heterogêneo e logo, a qualidade de vida na velhice é um fenômeno multidimensional e seus fatores determinantes são variáveis.

\subsection{Qualidade de vida}

A definição de qualidade de vida criada pela OMS no início da década de 90 diz que esta se refere à percepção do indivíduo sobre sua posição na vida no contexto cultural e no sistema de valores no qual ele vive, seus desejos e preocupações. Para Fleck, Lousada; Xavier, Chachamovich, Vieira, Santos e Pinzon (1999) a definição criada pela OMS deixa claro que o conceito qualidade de vida é subjetivo, multidimensional e inclui elementos positivos e negativos.

Sendo assim, avaliar qualidade de vida envolve critérios biológicos, psicológicos e sociais e depende de muitos elementos em interação constante ao longo da vida do idoso. Para Vecchia, Ruiz, Bocchi e Corrente (2005), este conceito está relacionado à autoestima e ao bem estar pessoal e abrange uma série de aspectos como condições físicas do ambiente e condições oferecidas pela sociedade, relativas à renda, saúde, educação, interação social, às condições biológicas derivadas da genética e ao estilo de vida.

Neri (2005c) afirma que um dos modelos mais explicativos sobre qualidade de vida na velhice foi desenvolvido por Lawton. Trata-se de um modelo estrutural que leva em conta fatores objetivos e subjetivos, adotando uma perspectiva de curso de vida. Seu modelo incide sobre quatro áreas sobrepostas das quais depende a funcionalidade do idoso: a) competência comportamental: consiste na avaliação do funcionamento do indivíduo em relação à saúde, à funcionalidade física, à cognição, ao comportamento social e à utilização do tempo. b) condições ambientais: refere-se à relação que o indivíduo estabelece com seu meio natural e à sua adaptação ao ambiente. As condições ambientais irão permitir ao idoso desempenhar comportamentos adaptativos e irão promover a qualidade de vida na velhice. c) qualidade de vida percebida: representa a avaliação subjetiva que cada pessoa faz sobre seu desempenho em qualquer domínio das competências comportamentais. d) bem estar subjetivo: refere-se a uma autoavaliação sobre a relação entre competência comportamental, condições objetivas e qualidade de vida percebida. É referenciado a dois tipos de indicadores, cognitivos e emocionais. O primeiro está relacionado à satisfação global com a vida e à satisfação referenciada a domínios (moradia, saúde física). O segundo diz respeito à avaliação de estados afetivos positivos (alegria) e negativos (tristeza).

Tendo em vista a necessidade de repensar políticas e práticas relacionadas à velhice, tornou-se inquestionável a importância do 
desenvolvimento de pesquisas que investiguem as variáveis físicas e psicológicas a fim de proporcionar uma melhor qualidade de vida aos idosos. Nesse sentido, objetivou-se com esta pesquisa identificar os comportamentos de saúde, o senso de controle e a qualidade de vida dos idosos residentes na zona urbana do município de Patos de Minas - MG.

\section{Método}

O presente trabalho foi desenvolvido a partir do banco de dados da pesquisa "Saúde, mecanismos de auto-regulação do self e qualidade de vida no envelhecimento normal: estudo com a população idosa de Patos de Minas - MG" aprovado pelo Comitê de ética em Pesquisa do Centro Universitário de Patos de Minas - UNIPAM (protocolo no 110/08). A pesquisa foi realizada nesta instituição, dentro da Faculdade de Ciências da Saúde, em conjunto com os alunos do curso de Psicologia participantes da "Liga de Psicogerontologia". Tratou-se de um estudo descritivo, de campo, quantitativo, de natureza transversal destinado a avaliar variáveis sociais, psicológicas e físicas de uma amostra da população idosa do município de Patos de MinasMG.

\subsection{Participantes}

O processo adotado para a determinação da amostra foi a técnica de amostragem probabilística aleatória simples para a construção de um subconjunto delimitador do universo adotado. $O$ resultado da amostra, utilizando-se o último censo demográfico, para um nível de confiança de $95 \%$, com um erro amostral tolerável de $5 \%$ e para um universo de 8494 idosos residentes no município de Patos de Minas, foi de 364 sujeitos. Como os idosos representam $4,27 \%$ da população, foram calculadas amostras estratificadas considerando-se o número de idosos residentes em cada bairro da cidade.

Considerou-se como critérios de inclusão na pesquisa ter idade igual ou superior a 60 anos, residir na comunidade (isto é, não estar institucionalizado), no município de Patos de Minas e atingir o escore mínimo esperado, em um total de 30 pontos, segundo a escolaridade no Mini-Exame do Estado Mental (a nota de corte deste instrumento é dada pela escolaridade, segundo os critérios determinados por Brucki, Nitrini, Caramelli, Bertolucci e Okamoto, 2003: para analfabetos, 19; para escolaridade de 1 a 4 anos, 23; de 5 a 8 anos, 26,5; de 9 a 11 anos, 28; para indivíduos com escolaridade superior a 11 anos, 29. Esta é a escala mais amplamente utilizada na avaliação de declínio cognitivo em idosos). 
Participaram do estudo 364 idosos, sendo que duzentos e quarenta e quatro $(67 \%)$ dos entrevistados era de mulheres e cento e vinte (33\%) de homens com a idade mínima de 60 e máxima de 93 anos de idade. A média de idade dos participantes foi de 68,96 anos com desvio padrão de 7,33. Em relação ao estado civil 54,4\% eram casados, $33,8 \%$ viúvos, $7,1 \%$ divorciados e apenas $4,7 \%$ eram solteiros.

Quanto à escolaridade dos participantes observou-se que $12,9 \%$ são analfabetos, $50,4 \%$ estudaram pelo menos de um a quatro anos, $18 \%$ de cinco a oito anos, $13 \%$ de nove a 11 anos, e $7 \%$ estudaram mais de 11 anos.

Em relação aos arranjos de moradia dos participantes, 26,9\% moram com cônjuge, filhos e/ou netos, $26,4 \%$ moram com o cônjuge, $22 \%$ moram com filhos e/ou netos, $17,9 \%$ moram sozinhos e $6,9 \%$ moram com outras pessoas. Grande parte dos entrevistados não exerce atividade remunerada $(77,5 \%)$, no entanto uma minoria de $22,5 \%$ afirmou trabalhar.

\subsection{Procedimentos}

a) Acesso à amostra: foi obtido pelos acadêmicos do curso de Psicologia participantes do projeto "Saúde, mecanismos de autoregulação do self e qualidade de vida no envelhecimento normal: estudo com a população idosa de Patos de Minas - MG". Os alunos foram nas residências nos bairros selecionados do município de Patos de Minas para identificar os domicílios que tinham um morador idoso e que preenchessem os critérios de inclusão na pesquisa.

b) Coleta de dados: A coleta dos dados foi feita no domicílio do idoso. Os voluntários foram informados acerca dos objetivos da pesquisa, sua opção individual em participar ou não, o sigilo da identidade e a importância em fornecer respostas sinceras para a validade do estudo. Após a resposta afirmativa do sujeito em colaborar, foi solicitado que assinasse o Termo de Consentimento Esclarecido. Em seguida, mediante entrevista, os instrumentos foram respondidos individualmente.

c) Análise dos dados: Os dados obtidos foram submetidos à análise estatística com a utilização do programa SPSS (Statistical Package for Social Sciences). Foram feitas tabelas de frequência para as variáveis categóricas, bem como estatísticas descritivas das variáveis contínuas (média e desvio padrão). A análise de regressão foi utilizada para verificar as variáveis que mais contribuem para a qualidade de vida dos idosos investigados.

\subsection{I nstrumentos}


a) Anamnese para caracterizar os participantes quanto à idade, sexo, estado civil, escolaridade, histórico de saúde, engajamento social, estilo de vida e hábitos de saúde, autopercepção da saúde física e mental, entrevista com o familiar para informações adicionais.

b) Escala de Lawton (BRASIL, 2006). Avalia o desempenho funcional em termos de atividades instrumentais e os idosos são classificados como independentes, dependentes parcialmente ou dependentes no desempenho de nove funções.

c) Mini-exame do Estado Mental (BRUCKI; NITRINI; CARAMELLI; BERTOLUCCI; OKAMOTO, 2003). É um teste de rastreamento e permite uma avaliação global do funcionamento cognitivo. Funciona como indicador de déficit cognitivo.

d) Escala de Crenças de Controle (FONTES, 2006), tipo Likert, variando de um (nunca) a quatro (frequentemente), dividida em duas subescalas (agência pessoal; agência interpessoal), sendo que agência pessoal é a possibilidade do individuo de exercer controle através de recursos próprios incluindo conhecimento, habilidade e esforço. Já a agência interpessoal trata de comportamentos como comunicação das necessidades e cooperação para com outros, visando alcançar os objetivos.

e) Escala Adaptada de Qualidade de Vida de Flanagan (DANTAS; GÓIS; SILVA, 2005). São avaliados os domínios "Bem estar físico e mental", "Relacionamento com outras pessoas", "Atividades cívicas, sociais e comunitárias", "Desenvolvimento e enriquecimento pessoal", "Recreação" e "independência".

\section{Resultados e discussão}

Sobre o desempenho funcional em termos das atividades instrumentais de vida diária, verificou-se que apenas $0,3 \%$ é dependente, $7,7 \%$ é parcialmente dependente e $92 \%$ é independente. Este resultado mostra-se bastante positivo uma vez que uma boa qualidade de vida na velhice relaciona-se com a autonomia e a independência na realização de atividades cotidianas. Segundo Rolim e Forti (2004), relaciona-se com o fato de eles poderem sentir-se melhor mediante conseguirem cumprir com suas funções diárias básicas adequadamente, e conseguirem viver de uma forma independente.

Quanto ao estilo de vida, os idosos foram questionados quanto ao engajamento em atividades sociais e à prática de comportamentos preventivos positivos para a saúde. A análise dos dados revelou que $78 \%$ não participa de nenhuma atividade na comunidade, seja ela social ou voluntária. Entre os idosos que participam, $60 \%$ o fazem pelo menos uma ou duas vezes por semana. A participação em alguma atividade religiosa é exercida por $70 \%$ dos participantes, 
sendo que a maioria (60\%) a exerce com a frequência de uma a duas vezes por semana.

A grande participação em alguma atividade religiosa foi notada. De acordo com Cardoso e Ferreira (2009) estudos têm mostrado que a religiosidade está associada ao bem estar psicológico, e aos aspectos que contribuem para o ajustamento na velhice, como as relações positivas com os outros, a autoaceitação, o crescimento pessoal e a satisfação com a vida. De uma maneira geral, as autoras afirmam que a religiosidade influencia positivamente o bem estar do idoso, pois interfere na percepção do mesmo sobre suas experiências aumentando seu senso de controle e segurança pessoal.

Quando questionados sobre a prática de exercícios físicos, verificouse que a maioria não tem uma participação regular: $42 \%$ declaram participar nunca ou raramente, $21 \%$ responderam que às vezes, outros $21 \%$ declaram praticar exercícios físicos pelo menos uma vez na semana e $16 \%$ praticam atividades físicas todos os dias. Quanto ao exame regular da pressão e do colesterol, $6 \%$ disseram examinar nunca ou raramente, $36 \%$ examinam às vezes e $58 \%$ examinam sempre. Sobre a vacinação, $92 \%$ afirmam manter as vacinas atualizadas. Visitas regulares ao médico são praticadas por $82 \%$ dos participantes. Destes, a data da última visita contada a partir da data da entrevista, tinha acontecido com $26 \%$ há um mês, $17 \%$ há dois meses, $12 \%$ há três meses, $8 \%$ há quatro meses, $24 \%$ de cinco a 11 meses, $8 \%$ há um ano e $5 \%$ apenas havia procurado há mais de um ano atrás.

Os cuidados relacionados à saúde física dos idosos de Patos de Minas chamaram atenção, pois a grande a maioria da amostra pesquisada examina a pressão e o colesterol regularmente, mantém as vacinas atualizadas e visita o médico regularmente. Atitudes como essas contribuem não só para a saúde física, mas segundo Rolim e Forti (2004) proporcionam melhoras nos aspectos psicológicos e sociais das pessoas que a exercem regularmente. No entanto, apesar do engajamento verificado em vários comportamentos preventivos, a atividade física não foi um deles. A prática regular de atividades físicas vem sendo apontada como uma variável importante para a promoção da saúde tanto física quanto cognitiva. Siqueira, Nahas, Facchini, Silveira, Piccini, Tomasi, Thumé, Reichert e Hallal (2009) analisaram os fatores que adultos e idosos consideram como mais importantes para manutenção da saúde. A amostra foi composta por 4.060 adultos e 4.003 idosos do Rio grande do Sul, Pernambuco, Santa Catarina e Alagoas. Os fatores mais indicados pelos adultos foram a alimentação saudável $(33,8 \%)$, a realização de exercício físico $(21,4 \%)$ e não fumar $(13,9 \%)$. Já os idosos afirmaram que era a alimentação saudável $(36,7 \%)$, não fumar $(17,7 \%)$ e consultar 0 médico regularmente $(14,2 \%)$ como fatores importantes para a manutenção da saúde. 
Quanto ao senso de controle, a média encontrada para a agência pessoal foi de 2,94 $(\mathrm{DP}=0,61)$ e de $2,96(\mathrm{DP}=0,61)$ para a agência interpessoal e agência total $(D P=0,68)$, indicando que com frequência, os participantes disseram ter crenças de controle pessoal e interpessoal sobre como lidar com as circunstâncias importantes de sua vida, numa escala variando de 1 =nunca, 2 =algumas vezes, $3=$ com frequência, $4=$ sempre.

Um estudo realizado por Fontes (2006) analisou o índice de consistência interna da Escala de Crenças de Controle para cada subescala, e também para escala total, onde os valores de alpha de Cronbach encontrados foram de $a=0,75$ para as crenças de agência, $a=0,74$ para as crenças de agência pessoal e $a=0,43$ para as crenças de agência interpessoal. Esses valores indicam que 0 instrumento tem consistência interna, com exceção da subescala de crenças de agência interpessoal que requer mais estudos para identificar quais pontos necessitam de mudanças.

Para Silva e Yu (2009) o senso de controle constitui tema relevante para melhor entender como as pessoas podem decidir sobre temas que afetam ou afetarão a manutenção de seu estilo de vida. Quanto ao senso de controle, neste estudo percebeu-se que os participantes exercem com frequência controle sobre a avaliação da agência pessoal e interpessoal. Isso quer dizer que os idosos da amostra pesquisada conseguem exercer certo controle sobre os eventos de suas vidas através de recursos próprios incluindo conhecimento, habilidade e esforço, e que ainda seus objetivos são alcançados mediante a agência interpessoal, ou seja, através de comportamentos como comunicação das necessidades e cooperação para com outros. Quanto maior o senso de controle pessoal, mais intensos são os sentimentos de satisfação e que, quanto menor, maiores as possibilidades de depressão, preocupação e desamparo.

A Escala Adaptada de Qualidade de Vida de Flanagan apresentou consistência interna neste estudo com um coeficiente $a=0,78$. Estudo realizado por Schmidt e Dantas (2006) o valor encontrado foi de $a=0,81$. Esses índices indicam que os instrumentos possuem consistência interna e, assim, garantem sua validade para utilização na presente pesquisa.

De modo geral $(M=5,05 ; D P=0,77)$, a maioria estava satisfeita $(57,4 \%)$ ou bastante/extremamente satisfeita $(24,7 \%)$ com sua qualidade de vida. O maior índice de satisfação apareceu no domínio 'Independência' $(83,2 \%)$ e a menor satisfação no domínio 'Recreação' $(59,1 \%)$.

Quanto ao domínio 'Bem estar físico e mental' ( $M=5,13 ; D P=0,95)$, $33 \%$ dos idosos estavam extremamente ou bastante satisfeitos, $45,1 \%$ satisfeitos, $17,7 \%$ nem satisfeitos nem insatisfeitos e $4,2 \%$ insatisfeitos. No que se refere ao 'Relacionamento com outras pessoas' $(M=5,20 ; \quad D P=1,06), \quad 41 \%$ dos idosos estavam 
extremamente ou bastante satisfeitos, $39 \%$ satisfeitos, $13,9 \%$ nem satisfeitos nem insatisfeitos, $5 \%$ insatisfeitos e 1,4\% bastante /extremamente insatisfeitos. No que tange às 'Atividades cívicas, sociais e comunitárias' $(M=4,92 ; \quad D P=0,99), 21,8 \%$ dos idosos estavam extremamente ou bastante satisfeitos, $44,8 \%$ satisfeitos, $26,2 \%$ nem satisfeitos nem insatisfeitos, $4,1 \%$ insatisfeitos e $1,1 \%$ bastante ou extremamente insatisfeitos. Com relação ao 'Desenvolvimento e enriquecimento pessoal' $(M=5,07$; $D P=0,86)$, $26,3 \%$ dos idosos estavam extremamente ou bastante satisfeitos, $54,9 \%$ satisfeitos, $15,8 \%$ nem satisfeitos nem insatisfeitos, $2,2 \%$ insatisfeitos e $0,9 \%$ bastante ou extremamente insatisfeitos. Quanto à 'Recreação' $(M=4,67 ; \quad D P=0,99), 16,6 \%$ dos idosos estavam extremamente ou bastante satisfeitos, $42,5 \%$ satisfeitos, $31,8 \%$ nem satisfeitos nem insatisfeitos, $7,5 \%$ insatisfeitos e $1,6 \%$ bastante ou extremamente insatisfeitos. Por último, em relação ao domínio 'Independência' $(M=5,39 ; \quad D P=1,15), 46,2 \%$ dos idosos estavam extremamente ou bastante satisfeitos, $37 \%$ satisfeitos, $10,8 \%$ nem satisfeitos nem insatisfeitos, $4,7 \%$ insatisfeitos e $1,4 \%$ bastante ou extremamente insatisfeitos.

Para a análise de regressão foi utilizado o método stepwise para testar quais as variáveis são preditoras da qualidade de vida. O resultado da regressão linear acusou uma causalidade, verificando que o senso de controle influencia na percepção de qualidade de vida $\left(r^{2}=0,54 ; p<0.01\right)$.

Os participantes da pesquisa estavam satisfeitos com a maioria dos domínios avaliados para a qualidade de vida. Entre eles o domínio de maior satisfação foi o de independência. Para Neri (2005a), quando os idosos gozam de autonomia funcional, eles próprios podem providenciar arranjos para que seu ambiente se torne mais seguro, variado e interessante. Dessa forma, os idosos por si mesmos são capazes de tomarem atitudes construindo um ambiente em que possam viver melhor.

É interessante notar que os participantes mostraram-se satisfeitos com o domínio relacionamento com outras pessoas, considerado importante, pois a capacidade de iniciar e manter contatos sociais influencia a percepção sobre a qualidade de vida e é um importante preditor de satisfação na velhice. No entanto, a grande maioria da amostra estudada não participa de nenhuma atividade na comunidade seja ela social ou voluntária e a avaliação dos domínios atividades cívicas e sociais, e recreação da escala de Flanagan apontaram os resultados como nem satisfeitos, nem insatisfeitos, o que não interferiu na percepção geral de satisfação na qualidade de vida. Esse fato pode ser explicado pela teoria da seletividade socioemocional de Laura L. Carstensen que segundo Neri, (2006b) explica o declínio nas interações sociais e as mudanças do comportamento emocional dos idosos. Segundo esta teoria, a 
redução da rede de relações sociais, e a participação social na velhice ocorrem devido a uma redistribuição ativa de recursos socioemocionais dos idosos, em virtude da mudança em sua perspectiva de futuro. Ou seja, na velhice as relações interpessoais mais próximas como família e amigos são mais importantes para a adaptação do idoso. Neste sentido, uma participação reduzida na comunidade, para esta amostra, não promoveu influências negativas na sua qualidade de vida.

Em consonância com essas práticas encontra-se a avaliação dos domínios bem estar físico e mental e desenvolvimento e enriquecimento pessoal, colocados como satisfeitos por grande parte dos participantes. De acordo com Ribeiro e Yassuda (2007) o estilo de vida escolhido pelas pessoas determina a qualidade de seu envelhecimento, pois a escolha dos alimentos ingeridos e as atividades ocupacionais, sociais e físicas influenciam a capacidade de evitar doenças, o nível e funcionamento físico e mental, e o engajamento com a vida. Essa perspectiva está baseada no pressuposto de que, pelo menos parcialmente os indivíduos podem controlar como envelhecerão, dentro das limitações impostas pelo contexto socioeconômico onde estão inseridos.

\section{Considerações finais}

Essa pesquisa teve como objetivo investigar os comportamentos de saúde, o senso de controle e a qualidade de vida dos idosos residentes na zona urbana do município de Patos de Minas - MG. Os resultados encontrados mostraram que estilo de vida exercido pelos participantes proporcionava qualidade de vida, visto que a amostra apresenta um conjunto de comportamentos de saúde que trazem benefícios não só para saúde física, mas também para o bem estar psicológico e o engajamento social. A grande maioria dos participantes era independente, permitindo que os mesmos tivessem mais autonomia, exercendo controle sobre sua própria vida. Em relação ao senso de controle, os idosos mostraram utilizar com frequência recursos próprios para alcançarem seus objetivos. Os três construtos investigados parecem estar interligados na amostra, onde o estilo de vida e a utilização de recursos próprios para o alcance de seus objetivos praticados pelos participantes promoveram uma boa avaliação sobre a sua qualidade de vida.

Acredita-se que este estudo traz contribuições não só aos idosos, a título de prevenção, mas aos serviços de saúde da área de gerontologia, na medida em que contribui com informações para os profissionais que cuidam da saúde do idoso, ampliando o banco também com informações dessa área de pesquisa, na busca de novos e melhores meios de lidar e cuidar dessa faixa etária da população 
que cresce consideravelmente a cada ano. Acredita-se ainda que novas pesquisas na área, e com amostras oriundas de outras realidades sociais, possam gerar mais informações sobre o comportamento do idoso e sobre a qualidade de vida. Essas informações são importantes tanto para a população idosa, quanto para os profissionais da área, pois quanto mais se conhece a respeito da população a qual se pretende assistir, levando em conta suas peculiaridades e limites e suas condições reais de atuação, melhor o atendimento e mais benefícios pode-se trazer para aquela população.

\section{Referências}

BRASIL. Ministério da saúde. Secretaria de atenção à saúde. Departamento de Atenção Básica. Envelhecimento e saúde da pessoa idosa. Brasília: Ministério da Saúde, Cadernos de Atenção Básica, n. 19, 2006. 192f.

BRUCKI, S. M. D; NITRINI, R; CARAMELLI, P; BERTOLUCCI, P. H. F; OKAMOTO, I. H. Sugestões para o uso do Mini-Exame do Estado Mental no Brasil. Arquivos de Neuropsiquiatria, São Paulo, v. 61, n. 3-B, p. 777-781, 2003.

CARDOSO, M. C. S.; FERREIRA, M. C. Envolvimento religioso e bem estar subjetivo em idosos. Psicologia: Ciência e Profissão, Brasília, v. 29, n. 2, p. 380-393, jun. 2009.

DANTAS, R. A. S.; GOIS, C. F. L.; SILVA, L. M. Utilização da versão adaptada da escala de qualidade de vida de Flanagan em pacientes cardíacos. Revista Latino-Americana de Enfermagem, Ribeirão Preto, v. 13, n. 1, fev. 2005.

FLECK, M. P. A; LOUSADA, S; XAVIER, M; CHACHAMOVICH, E; VIEIRA, G; SANTOS, L; PINZON, V. Aplicação da versão em português do instrumento de avaliação de qualidade de vida da Organização Mundial da Saúde (WHOQOL-100). Revista de Saúde Pública, São Paulo, v. 33, n. 2, p. 198-205, 1999.

FONTES, A. P. O enfrentamento do stress no trabalho na idade adulta. 2006. 132 f. Dissertação (Mestrado em Gerontologia) faculdade de Educação, Universidade Estadual de Campinas, Campinas.

GOLDSTEIN, L. L. No comando da própria vida: A importância de crenças e comportamentos de controle para o bem estar na velhice. In: NERI, A. L.; FREIRE, S. A. E por falar em boa velhice. Campinas, SP: Papirus, 2000, p. 55-67.

NERI, A. L. Crenças de auto-eficácia e envelhecimento bem-sucedido. In: FREITAS, E. V.; PY, L.; NERI, A. L.; CANÇADO, F. A. X.; GORZONI, M. L.; ROCHA, S. M. Tratado de Geriatria e Gerontologia. 2. ed. Rio de Janeiro: Guanabara Koogan, 2006 (a), p. 1267-1276. 
NERI, A. L. Teorias psicológicas do envelhecimento: percurso histórico e teorias atuais. In: FREITAS, E. V.; PY, L.; NERI, A. L.; CANÇADO, F. A. X.; GORZONI, M. L.; ROCHA, S. M. Tratado de Geriatria e Gerontologia. 2. ed. Rio de Janeiro: Guanabara Koogan, 2006 (b). p. 345-389.

NERI, A. L. Qualidade de vida na velhice e atendimento domiciliário. In: DUARTE, Y. A. O; DIOGO D'ELBOUX, M. J. Atendimento domiciliar: um enfoque gerontológico. São Paulo: Atheneu, 2005 (a). p. 33-47.

NERI, A. L. Qualidade de vida na velhice. In: NERI, A. L. (Org.). Palavras-chave em Gerontologia. 2. ed. Campinas, SP: Alínia, 2005 (b), p. 162-165.

NERI, A. L. Controle percebido. In: NERI, A. L. (Org.). Palavraschave em Gerontologia. 2.ed. Campinas, SP: Alínia, 2005(c). p. 37-40.

OLIVEIRA, S. F. D; DUARTE, Y. A. O; LEBRÃO, M. L; RUY, L. Demanda referida e auxílio recebido por idosos com declínio cognitivo no município de São Paulo. Saúde e Sociedade, São Paulo, v. 16, n. 1, p. 81-89, 2007.

PETROSKI, E. L.; PELEGRINI, A. Associação entre o estilo de vida dos pais e a composição corporal dos filhos adolescentes. Revista Paulista de Pediatria, São Paulo, v. 27, n. 1, p. 48-52, 2009.

RIBEIRO, P. C. C.; YASSUDA, M. S. Cognição, estilo de vida e qualidade de vida na velhice. In: NERI, A. L. (Org.). Qualidade de vida na velhice: enfoque multidisciplinar. Campinas, SP: Alínea, 2007, p. 189-284.

ROLIM, S. F.; FORTI; V. A. M. Envelhecimento e atividade física: auxiliando na melhoria e manutenção da qualidade de vida. In: DIOGO D'ELBOUX, M. J.; NERI, A. L.; CACHIONI, M. (Orgs.). Saúde e Qualidade de Vida na Velhice. Campinas, SP: Alínia, 2004. p. 57-73.

SILVA, W. M.; YU, A. S. O. Análise Empírica do Senso de Controle: Buscando Entender o Excesso de Confiança. Revista de Administração Contemporânea, Curitiba, v. 13, n. 2, p. 247-271, Abr./Jun. 2009.

SKAFF, M. M. Sense of control and health. In: ALDWIN, C. M; PARK, C. L; SPIRO III, A. Handbook of Health Psychology and Aging. New York: The Guilford Press, 2007. p. 186-209.

SCHMIDT, D. R. C, DANTAS, R. A. S. Qualidade de vida entre profissionais de enfermagem do bloco cirúrgico. Revista Gaúcha de Enfermagem, Porto Alegre, v. 27, n. 1, p. 100-108, mar. 2006.

SIQUEIRA, F. V.; NAHAS, M. V.; FACCHINI, L. A.; SILVEIRA, D. S.; PICCINI, R. X.; TOMASI, E.; THUMÉ, E.; REICHERT, F. F.; HALLAL, P. R. C. Fatores considerados pela população como mais importantes para manutenção da saúde. Revista Saúde Pública, v. 43, n. 6, p. 961-71, 2009. 
STRAUB. R. O. (Org.). Permanecendo saudável. In: STRAUB, R. O. Psicologia da Saúde. Porto Alegre: Artmed, 2005. p. 193-230.

VECCHIA, R. D; RUIZ, T; BOCCHI, S. C. M; CORRENTE, J. E. Qualidade de vida na terceira idade: um conceito subjetivo. Revista Brasileira de Epidemiologia, São Paulo, v. 8, n. 3, p. 246-252, set. 2005.

WROSCH, C.; SCHULZ, R. Health-engagement control strategies and 2-year changes in older adults' physical health. Psychological Science, v. 19, n. 6, p. 537-541, 2008.

\section{Endereço para correspondência Lourine Severo Oliveira}

Av Brasil, no 45 centro. Niquelândia Goiás. Cep: 76420-000.

Endereço eletrônico: lourine.psico@hotmail.com

\section{Dóris Firmino Rabelo}

Centro de Ciências da Saúde da Universidade Federal do Recôncavo da Bahia. Rua do Cajueiro s/n Bairro Cajueiro. Santo Antônio de Jesus - BA. CEP: 44574-490.

Endereço eletrônico: drisrabelo@yahoo.com.br

Nelma Caires Queroz

Centro Universitário de Patos de Minas- Unipam. Rua Major Gote, 808 - Bairro Caiçaras. Patos de Minas- MG. CEP: 38702-054.

Endereço eletrônico: nelmacaires@yahoo.com.br

Recebido em: 09/09/2010

Reformulado em: 16/03/2011

Aceito para publicação em: 18/07/2011

Acompanhamento do processo editorial: Eleonôra Torres Prestrelo

\section{Notas}

* Psicóloga pela Faculdade de Ciências da Saúde, do Centro Universitário de Patos de Minas-UNIPAM.

**Psicóloga, Mestre em Gerontologia pela Unicamp e Docente do curso de Psicologia da Universidade Federal do Recôncavo da Bahia - UFRB.

*** Psicóloga, Mestre em Gerontologia e Doutora em Educação pela Unicamp. Docente do curso de Psicologia da Faculdade Ciências da Saúde pelo Centro Universitário de Patos de Minas - UNIPAM. 